\title{
Detección y descripción de rasgos psicopáticos en adolescentes utilizando el MACI ${ }^{*}$
}

\author{
Screening and describing psychopathic traits \\ in adolescents using the MACI
}

Recibido: abril 14 de 2010 | Revisado: junio 23 de 2010 | Aceptado: agosto 8 de 2010

\author{
EUGENIA V. VINET ** \\ Paula Alarcón Bañares \\ Ricardo Pérez-Luco Arenas \\ Departamento de Psicología, Universidad de La Frontera,
}

Temuco, Chile

Para citar este artículo. Vinet, E. V., Alarcón, B. P. \& Pérez-Luco Arenas, R. (2011). Detección y descripción de rasgos psicopáticos en adolescentes utilizando el MACI. Universitas Psychologica, 10 (3), 705-719.

* Artículo de investigación desarrollado con financiamiento del Proyecto del Fondo Nacional de Desarrollo Científico y Tecnológico del Gobierno de Chile (FONDECYT) № 1070397, titulado "Caracterización y evaluación multidimensional de adolescentes con desadaptación social".

*** Departamento de Psicología, Universidad de La Frontera, Temuco, Chile. Correos electrónicos: evinet@ufro.cl,paulandr@ufro.cl, perez-luco@ ufro.cl

\section{RES UMEN}

Este estudio reporta las características psicométricas de las dos escalas de Psicopatía del MACI y evalúa su utilidad para pesquisar y describir a adolescentes con alta psicopatía. Cuatro grupos representantes del continuo adaptación-desadaptación social respondieron el MACI y un autorreporte de comportamiento social adolescente. El análisis factorial de las escalas determinó estructuras de dos y tres factores, respectivamente, que son respaldadas por los modelos de psicopatía de Hare y de Cookie y Michie. Un algoritmo desarrollado con las puntuaciones de estas escalas identificó y permitió caracterizar un grupo de 49 jóvenes (11.2 \% de la muestra) con alta psicopatía. Se discuten las características psicométricas y la utilidad de estas escalas para identificar a adolescentes con perfiles psicopáticos.

Palabras clave autores

Escalas de Psicopatía, adolescentes, MACI, evaluación.

Palabras clave descriptores

Pruebas psicológicas, psicometría, investigación cuantitativa, análisis factorial.

\section{A B S T R A C T}

This study reports psychometric characteristics for two Psychopathy scales developed with the MACI and assess their utility for screening and describing high psychopathic adolescents. Four groups representing the social adjustment and maladjustment continue answered the MACI and a self report about adolescent social behaviour. A factorial analysis of the psychopathy scales determined structures formed by two and three factors which are supported by the models of psychopathy presented by Hare and by Cookie and Michie. An algorithm developed with these scales-scores identified and allowed to characterize a group of 49 adolescents $(11.2 \%$ of the sample) with high psychopathy. Psychometric characteristics and the utility of these two scales for identifying adolescents with psychopathic profiles are discussed.

Key words authors

Psychopathy scales, adolescents, MACI, assessment.

Key words plus

Psychological test, psychometry, quantitative research, factor analysis. 


\section{Antecedentes}

La delincuencia es un fenómeno que presenta una alta prevalencia durante la adolescencia, siendo un período crítico para el inicio de comportamientos transgresores o antisociales. Los estudios sobre delincuencia juvenil muestran que: (a) los varones presentan mayor riesgo de conductas trasgresoras que las mujeres en una razón de 3 a 1, (b) la adolescencia es el período donde se comete el mayor número de comportamientos antisociales, (c) estos comportamientos tienden a disminuir hacia la adultez, (d) la aparición temprana de conductas trasgresoras es un buen predictor de la delincuencia en la adultez y (e) solo una pequeña proporción de adolescentes mantiene sus comportamientos transgresores en la adultez, pese a vivir en entornos de alto riesgo criminogénico (Farrington, 1996; Moffit, Caspi, Harrington \& Milne, 2002; Rutter, Giller \& Hagell, 2000, entre otros). Una línea de investigación que se ha abocado a estudiar el fenómeno de la delincuencia juvenil, utiliza el constructo de psicopatía infanto-juvenil, el cual prioriza las características personales que se presentan junto a la conducta trasgresora, por sobre otros aspectos (Forth, Kosson \& Hare, 2003; Frick, Barry \& Bodin, 2000; Frick \& Ellis, 1999; Frick, O’Brien, Wooton \& McBurnett, 1994). Otra línea de investigación está focalizada en las trayectorias delictivas, dando mayor relevancia a las variables del medio en que están inmersos los jóvenes que delinquen (Piquero, Farrington \& Blumstein, 2007). Este estudio se inserta preferentemente en la primera línea de investigación.

La psicopatía es un constructo polémico, de gran relevancia clínica y criminológica, usado para designar a personas adultas con un trastorno antisocial severo, crónico y difícil de tratar, el cual consta de dos facetas conceptualizadas por Hare (2003): una incluye características personales y afectivas, y está integrada por rasgos de egocentrismo, falta de sinceridad, insensibilidad y falta de remordimientos; la otra faceta incluye aspectos conductuales que se manifiestan en un estilo de vida desviado, con conductas antisociales que incluyen alta tasa de delitos, alta probabilidad de delitos violentos, alta proporción de reincidencia y mala respuesta al tratamiento. Una conceptualización alternativa (Cooke \& Michie, 2001) incluye tres dimensiones que abarcan: lo interpersonal, a través de un estilo arrogante, engañoso y artificioso, con rasgos de locuacidad, encanto superficial, egocentrismo y manipulación; lo afectivo, expresado en escaso remordimiento y culpa, crueldad, baja empatía, afectos superficiales y fracaso en aceptar la propia responsabilidad por las acciones y lo impulsivo, expresado en un estilo conductual irresponsable que incluye aburrimiento, búsqueda de la estimulación, carencia de metas a largo plazo, impulsividad, ausencia de reflexión antes de actuar y un estilo de vida parasitario.

La psicopatía infanto-juvenil es un concepto resistido por la supuesta inmodificabilidad de las características involucradas en su definición; sin embargo, es un concepto importante para designar a ciertos niños y jóvenes que, junto a las conductas trasgresoras, presentan características personales y afectivas que, al igual que en los adultos, se expresan en egocentrismo, frialdad emocional, falta de sinceridad y de remordimientos (Forth et al., 2003; Frick et al., 2000; Frick \& Ellis, 1999; Frick, et al., 1994). La psicopatía infanto-juvenil tiene sus orígenes en el Trastorno de Conducta (TC), categoría diagnóstica que agrupa múltiples tipos de conducta, trayectorias y etiologías diferentes y que designa patrones de comportamiento antisocial persistentes y extremos para el nivel evolutivo del niño, los cuales entran en conflicto con las normas y los derechos o necesidades de los demás (Romero, 2001). El TC incluye las características conductuales, interpersonales y afectivas de la psicopatía y distingue dos subgrupos de niños, según la edad de inicio del trastorno (infantil o adolescente). El inicio temprano describe una trayectoria evolutiva más persistente y de mayor riesgo de consolidar una psicopatía en la adultez; el inicio en la adolescencia representaría una forma transitoria de enfrentar las demandas madurativas de esa etapa del desarrollo (Moffitt, 1993).

En la psicopatía infanto-juvenil se establece un vínculo entre el TC y la hiperactividad, planteándose que los niños con comportamiento impulsivo/ 
hiperactivo y problemas de conducta presentan mayor riesgo de conductas antisociales severas y persistentes que aquellos niños que solo presentan una de las dos condiciones (Lynam, 1996). En otra postura, Frick et al. (2000) restringen el concepto a un subgrupo pequeño de niños con trastornos de conducta e impulsividad/hiperactividad que presentan rasgos de crueldad y frialdad emocional o insensibilidad, semejantes a los descritos en la psicopatía adulta a través del componente afectivo de las conceptualizaciones de Hare (2003) y de Cooke y Michie (2001).

Psicométricamente, Frick et al. (1994) proponen una estructura de dos dimensiones en la pesquisa de la psicopatía, denominadas Impulsividad/Problemas de Conducta (I/PC) y Dureza/Insensibilidad emocional (D/I) las que abarcan los aspectos conductuales y afectivo/interpersonales del trastorno. Sugieren que el factor I/PC captura un constructo muy similar a las definiciones tradicionales de problemas de conducta, en tanto que valores altos en el factor D/I permiten identificar a un subgrupo de niños con problemas de conducta que tienen características especiales relacionadas con falta de sentimientos de culpa, emociones superficiales y falta de empatía, que actualmente se asocian a la conceptualización de la psicopatía infanto-juvenil.

Para evaluar estos adolescentes, y particularmente a aquéllos que infringen la ley, se han utilizado modelos multidimensionales que incluyen factores individuales, familiares y comunitarios de alto riesgo criminogénico. En estos modelos, los factores individuales han sido evaluados a través de escalas de personalidad, o bien, a través de escalas específicas de psicopatía. Entre las escalas de personalidad destaca el Inventario Clínico para Adolescentes de Millon (MACI) de 1993, en tanto que en la evaluación de características psicopáticas en la adolescencia el instrumento per se es la Psychopathy Checklist: Youth Version (PCL:YV) de Forth et al. (2003), la cual es una adaptación de la PCL-R de adultos (Hare, 2003) que evalúa rasgos psicopáticos en adolescentes entre 12 y 18 años, a través de una exhaustiva entrevista clínica semiestructurada e información procedente de registros externos.
Debido a la alta inversión profesional que demanda la PCL:YV, Murrie y Cornell (2000) y Salekin, Ziegler, Larrea, Anthony y Bennett (2003), proponen la creación de dos escalas de Psicopatía, a partir de ítems del MACI. La primera, denominada Psychopathy Content Scale (PCS) de Murrie y Cornell (2000), está constituida por 20 ítems que se relacionan conceptualmente con el constructo bidimensional de psicopatía de Hare (2003); la segunda escala, denominada Psychopathy-16 (P16) de Salekin et al. (2003), está compuesta por 16 ítems que se alinean con los planteamientos de Cooke y Michie (2001), representando las características de dureza/insensibilidad, egocentrismo y conducta antisocial. Los estudiosos de la delincuencia juvenil destacan que se trata de escalas breves que se aplican en la modalidad de autorreporte y que parecen evaluar adecuadamente el constructo de psicopatía en adolescentes. La PCS ha presentado buenas correlaciones con los dos factores de psicopatía de Hare y una capacidad de discriminación del $83 \%$ con respecto a alta y baja psicopatía, clasificada según la PCL-R (Murrie \& Cornell, 2000). Por otro lado, Salekin et al. (2003) documentan la buena capacidad de la P-16 para predecir reincidencia general y reincidencia violenta como escala total y a partir de sus facetas conducta antisocial y dureza/insensibilidad, siendo esta última la responsable de la reincidencia general y de las transgresiones violentas por parte de los jóvenes. Además, ambas escalas han sido sometidas a análisis factorial en jóvenes estadounidenses con trastornos conductuales severos (Penney, Moretti \& Da Silva, 2008), obteniéndose para la PCS una solución óptima de dos factores, compatible con el modelo de Hare, y para la P-16 una la solución de tres factores que se asemejan a la propuesta de Cooke y Michie (2001).

En Chile, país donde se desarrolla este estudio, el MACI ha sido validado para el trabajo con diferentes grupos de adolescentes, incluidos los infractores de ley (Alarcón, Vinet \& Salvo, 2005; Vinet \& Alarcón, 2003, 2009). Las dos escalas de Psicopatía del test se han utilizado exploratoriamente en el contexto de una evaluación multidimensional de jóvenes infractores de ley (Alarcón, Vinet, Salvo 
\& Pérez-Luco, 2009) y en la evaluación específica de la psicopatía realizada con el PCL:YV (Zúñiga, Vinet \& León, 2011). En ambos casos su aplicación ha resultado promisoria, despertando la necesidad de dedicarles un estudio específico, considerando el amplio uso que tiene el MACI en el trabajo con adolescentes infractores de ley y la necesidad de evaluar el constructo de psicopatía en los jóvenes delincuentes, para aportar a la distinción de grupos con características personales que ameriten tratamientos diferenciados.

Consecuentemente, este estudio aborda el concepto de psicopatía infanto-juvenil y se propone como objetivos: (a) evaluar el funcionamiento psicométrico de las escalas de Psicopatía del MACI en una muestra amplia de adolescentes chilenos representantes del continuo adaptación socialdesadaptación social; (b) determinar la estructura factorial de estas escalas en jóvenes chilenos; (c) evaluar empíricamente sus dimensiones factoriales como subescalas idóneas para la pesquisa de rasgos psicopáticos en adolescentes y (d) describir psicológicamente a los jóvenes que presentan altas puntuaciones en estas escalas de Psicopatía.

\section{Método}

\section{Participantes}

A partir de una muestra representativa de 901 escolares varones de la región centro-sur de Chile y una muestra de 264 adolescentes varones infractores de ley, procedentes de centros de atención ubicados en la misma región, se definió por conveniencia la muestra específica para este estudio. Incluyó 436 adolescentes entre 14 y 21 años de edad $(\mathrm{M}=16.7 ; \mathrm{DT}=1.48)$ que cubren el continuo adaptación social-desadaptación social, a través de cuatro grupos de jóvenes que fueron denominados Escolares con Baja Trasgresión social autorreportada (E-BT), Escolares con Alta Trasgresión social autorreportada (E-AT), Adolescentes Infractores de Inicio (AI-IN) y Adolescentes Infractores Reincidentes (AI-RE).

Los criterios de inclusión para definir los grupos fueron los siguientes: (a) E-BT: escolares con una puntuación total inferior a la mediana de toda la muestra, en la escala Conducta Antisocial Total (CAT) y puntaje 0 en la escala Delitos (D) de un cuestionario de autorreporte de conducta social; este criterio seleccionó a 124 sujetos del extremo inferior de la distribución de escolares. (b) E-AT: escolares con puntajes en la escala CAT y la escala D superiores a las medianas más una desviación intercuartil; este criterio seleccionó a 125 sujetos del extremo superior de la distribución de escolares. (c) AI-IN: adolescentes que no presentan delitos reiterados sancionados oficialmente por el sistema judicial y que en el autorreporte (escala D) señalan 2 o más delitos; este grupo quedó constituido por 95 adolescentes infractores. (d) AI-RE: adolescentes que habían sido sancionados por el sistema judicial por al menos tres delitos antes del delito actual, o que habían sido sancionados por al menos un delito y tenían tres delitos en proceso actual; este criterio seleccionó a 94 adolescentes infractores. Tanto en los AI-IN como en los AI-RE el criterio externo de categorización fue el registro SENAINFO del Servicio Nacional de Menores de Chile, el cual compila los antecedentes judiciales de los adolescentes sancionados por el sistema judicial chileno. Las características de los cuatro grupos, en función de las variables en estudio, se presentan en la primera parte de los resultados.

\section{Instrumentos}

Inventario Clínico para Adolescentes de Millon (MACI; Millon, 1993)

Este es un instrumento de construcción racional que sigue los modelos del aprendizaje biosocial y evolutivo de la personalidad, desarrollados por Millon (1969, 1990). Consta de 160 ítems con formato verdadero-falso que se organizan en 31 escalas, 27 de ellas con significado clínico las cuales evalúan Patrones de la Personalidad (12 escalas), Preocupaciones Expresadas (ocho escalas) y Síndromes Clínicos (siete escalas). Los estilos o patrones de personalidad son concebidos como modos de funcionamiento psicológico relativa- 
mente estables que surgen a través del desarrollo infantil y se estabilizan en la adolescencia como precursores del estilo de personalidad adulta. Las preocupaciones psicológicas se relacionan con aspectos significativos del desarrollo del adolescente e incluyen características del desarrollo de la identidad, corporalidad, sexualidad y relaciones sociales con los pares, familia y sociedad mayor. El área psicopatológica incluye los trastornos afectivos y de la adaptación social que tienen mayor prevalencia en la adolescencia. Las escalas restantes son tres escalas de control y una escala de validez de solo dos ítems.

Las escalas de Psicopatía, surgidas con posterioridad, son (1) la escala de Contenido Psicopático (PCS; Murrie \& Cornell, 2000) que consta de 20 ítems agrupados conceptualmente en tres facetas: Egocentrismo, Conducta antisocial y Abuso de sustancias y (2) la escala Psicopatía de 16 ítems (P-16; Salekin et al., 2003) estructurada racionalmente en tres facetas: Dureza/insensibilidad, Egocentrismo y Conducta antisocial.

El MACI es un instrumento multidimensional utilizado para describir el funcionamiento psicológico individual y de diferentes grupos de adolescentes; tiene características psicométricas respaldadas en estudios de diferentes países, entre los estudios chilenos están los de Vinet y Alarcón (2003) y Alarcón et al. (2005). Cuenta con normas estadounidenses, españolas y chilenas; estas últimas se presentan en puntajes transformados con puntos de corte (Vinet \& Forns, 2008) y acogen diferencias atribuidas a la cultura, observadas en adolescentes de origen latino (Vinet, 2010). Las escalas de Psicopatía han presentado niveles de confiabilidad superiores a 0.85 en adolescentes infractores o con trastornos psiquiátricos no psicóticos, y validez concurrente moderada con los instrumentos de Hare que evalúan específicamente el constructo de psicopatía (Murrie \& Cornell, 2000; Salekin et al., 2003), además presentan estructuras factoriales relativamente concordantes con las conceptualizaciones de psicopatía de Hare, en el caso de la PCS, y de Cooke y Michie en el caso de la P-16 (Penney et al., 2008).
Cuestionario de Autorreporte de Comportamiento Social Adolescente (CACSA; Alarcón, PérezLuco, Salvo, Roa, Jaramillo \& Sanhueza (2010)

Este es un instrumento construido racionalmente para explorar comportamientos prosociales y antisociales a través del registro de la frecuencia de conductas expresadas en una escala Likert de $0 \mathrm{a}$ 6 puntos (nunca $=0 ; 1$ ó 2 veces $=1$, varias veces $=3$, casi siempre $=5$ y siempre $=6$ ), consignando también, de modo dicotómico, la realización de esas conductas en los últimos 12 meses. Tiene 55 ítems que miden tres escalas principales: Comportamiento Prosocial (CPRO), Comportamiento Antisocial Total (CAT) y Abuso de Poder entre Pares (APP). La escala CAT posee seis subescalas: transgresiones sociales leves (TSL), trasgresión contra la propiedad (TPRO), trasgresión contra las personas (TPER), delitos (D) y consumo de alcohol y drogas (OHD); la escala APP tiene como subescala el ser víctima de abuso de poder (VAP).

El estudio psicométrico del CACSA (Alarcón et al., 2010), realizado diferenciadamente en muestras de escolares e infractores, señala índices de consistencia interna entre 0.86 (CAT) y 0.56 (APP) en los escolares $(\mathrm{Md}=0.725)$ y entre 0.90 (CAT) y 0.64 (APP) en los adolescentes infractores $(\mathrm{Md}=0.765)$. La estabilidad, evaluada en escolares, alcanza a 0.82 para CAT y 0.89 para CPRO. Con respecto a validez, el CACSA posee una capacidad para discriminar entre adolescentes escolares e infractores de ley, entre hombres y mujeres, y al interior de grupos de trasgresores que es compatible con lo establecido teóricamente. Además, la validez de contenido de las escalas del CACSA está respaldada por su relación con escalas e instrumentos que miden constructos asociados a la conducta antisocial; entre ellas se reportan relaciones positivas altamente significativas $(p<$ 0.001) entre la escala CAT y las escalas del MACI Transgresor y Predisposición Delictual tanto en escolares $(r=0.59 \mathrm{y} r=0.46$ respectivamente) como en infractores $(r=0.54 \mathrm{y} r=0.37$, respectivamente). Los autores concluyen que el CACSA posee capacidad para discriminar entre adolescentes con 
y sin delitos oficiales y entre grupos que, desde un punto de vista teórico, se espera presenten distintos niveles de comportamiento antisocial y prosocial. Como se ha señalado, las escalas CAT y D se utilizaron para crear los grupos E-BT y E-AT.

\section{Procedimiento}

Los escolares fueron contactados en sus colegios, realizando la evaluación a través de los gruposcurso. Los infractores fueron contactados en las instituciones donde estaban cumpliendo sus sanciones por sentencia judicial, siendo evaluados individualmente por los profesionales de los propios centros. En ambos casos, los adolescentes fueron informados de los objetivos de la investigación como "una forma de obtener mayor conocimiento de los jóvenes chilenos y sus experiencias asociadas a problemas de adaptación social"; además, se les planteó que la participación era voluntaria y se les aseguró la confidencialidad de la información; quienes aceptaron participar firmaron un documento de consentimiento informado.

\section{Análisis de Datos}

Los puntajes individuales del MACI y el CACSA de los cuatro grupos de la muestra de estudio, permitieron obtener los estadísticos descriptivos de cada grupo; en esta etapa también se obtuvieron los índices de confiabilidad por consistencia interna para las dos escalas de Psicopatía y sus facetas de contenidos. Posteriormente, se realizaron comparaciones de grupos a través de ANOVAS con pruebas post hoc de Tukey, considerando especialmente las escalas del CACSA y las escalas de Psicopatía del MACI. Seguidamente, con los puntajes de la muestra total en cada escala de psicopatía del MACI, se realizó un análisis factorial exploratorio a través de componentes principales con rotación Oblimin, interpretando los factores obtenidos según el contenido de los ítems. A continuación, se generaron las escalas factoriales con los ítems que integraban cada factor; se examinó su consistencia interna, se calcularon sus estadísticos descriptivos y se realizaron contrastes según grupo. Por último, se seleccionaron los sujetos con puntajes altos en las escalas factoriales más relevantes al constructo de psicopatía, y se evaluaron cuantitativa y cualitativamente según sus características específicas en el CACSA (conducta antisocial) y en el MACI (funcionamiento psicológico), para afinar interpretativamente la evaluación de psicopatía y sus dimensiones a partir del autorreporte.

\section{Resultados}

\section{Descripción general de la muestra}

La muestra total, conformada por 436 adolescentes varones pertenecientes a cuatro grupos diferenciados por su nivel de adaptación social, presentó una media de edad de 17.02 años $(D T=1.36)$ y una escolaridad media de 9.53 años de educación formal $(D T=1.72)$. Los valores medios de cada grupo se presentan en la Tabla 1 junto a los valores medios en las escalas del CACSA y en las escalas de psicopatía PCS y P-16, con sus respectivas facetas de contenido.

En esta tabla se observa que existe un ordenamiento entre los grupos con respecto a la conducta antisocial medida a través de tres escalas del CACSA (CAT, D, TNE) y también según las facetas de conducta antisocial de las escalas de Psicopatía. Este ordenamiento se altera en las otras escalas ya que el grupo de escolares con alta trasgresión autorreportada (E-AT) tiende a puntuar de modo similar a los infractores de inicio y, en algunas variables, a los infractores reincidentes, tanto en las escalas del CACSA como en la evaluación de psicopatía.

Los valores alfa calculados para las escalas de Psicopatía son 0.83 para la PCS y 0.69 para la P-16; los valores alfa de las facetas de contenido disminuyen a $0.52,0.54$ y 0.49 en las facetas de la PCS y а $0.48,0.54$ y 0.61 para las facetas de la P-16. 


\section{TABLA 1}

Estadísticos descriptivos y comparación de grupos en variables sociodemográficas, del CACSA y escalas de Psicopatía del MACI

\begin{tabular}{|c|c|c|c|c|c|c|c|c|c|}
\hline \multirow{2}{*}{ Variables } & \multicolumn{2}{|c|}{ E-BT } & \multicolumn{2}{|c|}{ E-AT } & \multicolumn{2}{|c|}{ AI-IN } & \multicolumn{2}{|c|}{ AI-RE } & \multirow{2}{*}{$F$} \\
\hline & M & DT & M & DT & M & DT & M & DT & \\
\hline Edad & $16.21_{\mathrm{a}}$ & 1.25 & $16.92_{\mathrm{b}}$ & 1.19 & $17.25_{b}$ & 1.18 & $17.94_{c}$ & 1.22 & $37.41 * * *$ \\
\hline Escolaridad & $10.17_{\mathrm{a}}$ & 1.09 & $10.59 \mathrm{a}$ & 1.02 & $8.74_{b}$ & 1.59 & $8.12_{c}$ & 1.95 & $72.94 * * *$ \\
\hline \multicolumn{10}{|l|}{ Escalas CACSA } \\
\hline Comportamiento antisocial total & $3.27 \mathrm{a}$ & 2.47 & $41.37_{b}$ & 19.15 & $33.44_{c}$ & 22.65 & $60.72_{d}$ & 25.94 & $175.45 * * *$ \\
\hline Trasgresiones sociales leves & $2.44_{a}$ & 2.28 & $16.81_{b}$ & 6.77 & $13.01_{c}$ & 7.84 & $18.72_{b}$ & 9.42 & $131.53 * * *$ \\
\hline Trasgresiones contra las personas & $0.82_{\mathrm{a}}$ & 1.40 & $13.76_{b}$ & 9.85 & $8.28_{c}$ & 7.52 & $13.31_{b}$ & 9.42 & $72.22 * * *$ \\
\hline Trasgresiones contra la propiedad & $0.15_{\mathrm{a}}$ & 0.38 & $7.72_{b}$ & 5.58 & $6.36_{b}$ & 5.81 & $12.85_{\mathrm{c}}$ & 6.65 & $116.34 * * *$ \\
\hline Delitos & $0.000_{a}$ & 0.00 & $13.05_{b}$ & 7.54 & $9.53_{\mathrm{c}}$ & 8.44 & $19.59 \mathrm{~d}$ & 9.05 & $150.44 * * *$ \\
\hline Consumo de drogas & $0.46_{a}$ & 1.05 & $8.46_{b}$ & 6.13 & $8.13_{\mathrm{b}}$ & 6.62 & $17.21_{c}$ & 7.83 & $149.19 * * *$ \\
\hline Trasgresión de normas para la edad & $0.56_{a}$ & 1.01 & $8.15_{b}$ & 4.47 & $12.00_{c}$ & 6.69 & $16.34_{d}$ & 7.69 & $173.37 * * *$ \\
\hline Comportamiento prosocial & $31.09 \mathrm{a}$ & 13.09 & 32.64 & 13.39 & $26.53_{\mathrm{b}}$ & 11.75 & $24.86_{b}$ & 10.67 & $9.41 * * *$ \\
\hline \multicolumn{10}{|l|}{ Escalas de Psicopatía } \\
\hline PCS total & $3.37_{\mathrm{a}}$ & 2.17 & $9.19_{b}$ & 3.26 & $8.75_{b}$ & 4.05 & $12.03 \mathrm{c}$ & 3.84 & $132.68 * * *$ \\
\hline Egocentrismo & $1.81_{\mathrm{a}}$ & 1.14 & $3.38_{b}$ & 1.74 & $2.66_{c}$ & 1.80 & $3.16_{b c}$ & 1.82 & $21.91 * * *$ \\
\hline Conducta antisocial & $1.02 \mathrm{a}$ & 0.87 & $2.48_{b}$ & 1.30 & $3.05_{c}$ & 1.13 & $3.48_{d}$ & 1.03 & $106.74 * * *$ \\
\hline Abuso de sustancias & $1.90_{\mathrm{a}}$ & 0.91 & $3.76_{b}$ & 1.36 & $3.39_{b}$ & 1.65 & $4.71_{c}$ & 1.32 & $87.80 * * *$ \\
\hline P-16 total & $3.58_{a}$ & 1.99 & $7.43_{b}$ & 2.49 & $7.28_{b}$ & 2.70 & $8.41_{c}$ & 2.99 & $81.65 * * *$ \\
\hline Dureza/insensibilidad & $0.86_{a}$ & 1.01 & $2.03_{b}$ & 1.31 & $1.41_{c}$ & 1.05 & $1.70_{b c}$ & 1.30 & $21.72 * * *$ \\
\hline Egocentrismo & $1.52_{\mathrm{a}}$ & 1.21 & $2.65_{b}$ & 1.44 & $2.35_{b}$ & 1.60 & $2.72_{b}$ & 1.74 & $16.11 * * *$ \\
\hline Conducta antisocial & $1.19 \mathrm{a}$ & 1.01 & $2.75_{b}$ & 1.34 & $3.53_{\mathrm{c}}$ & 1.01 & $3.99_{d}$ & 0.97 & $137.12 * * *$ \\
\hline
\end{tabular}

Nota: E-BT: Escolares con baja trasgresión autorreportada; E-AT: Escolares con alta trasgresión autorreportada;

AI-IN: Adolescentes infractores de inicio; AI-RE: Adolescentes infractores reincidentes.

Los grupos que tienen el mismo subíndice no presentan diferencias significativas entre sí.

$* * * p<0.001$

Fuente: elaboración propia.

\section{Análisis factorial exploratorio de las escalas de Psicopatía}

\section{Escala de Contenido Psicopático (PCS)}

La factorización de la escala PCS, utilizando el método de extracción por componentes principales con rotación Oblimin, entrega una estructura de cinco factores con autovalores mayores a 1.0 que explica el $50.9 \%$ de la varianza. Los primeros dos factores agrupan a 16 de los 20 ítems, el tercer factor agrupa a solo a dos de ellos (ítems 5 y 9) y presenta correlaciones negativas con los factores 4 y 5 . Ante estos resultados, se exploran soluciones alternativas observándose que la estructura de dos factores, que explica el $34.1 \%$ de la varianza, 
es la más coherente teóricamente, aunque el ítem 62 (Disfruto pensando en sexo) no carga significativamente en ninguno de los dos factores. Esta estructura presenta una correlación moderada entre los factores $(r=0.33)$ y tal como se aprecia en la Tabla 2, representaría las dos dimensiones básicas del modelo de Hare (2003). El primer com- ponente agrupa ítems sobre consumo de drogas y conducta antisocial, alineándose con la dimensión de conducta antisocial y estilo de vida desviado; el segundo componente agrupa ítems relacionados con frialdad emocional y manipulación interpersonal que corresponden a la dimensión afectiva interpersonal de este modelo.

\section{TABLA 2}

Estructura factorial de la escala de Contenido Psicopático en adolescentes chilenos

\begin{tabular}{|c|c|c|c|}
\hline \multirow{2}{*}{$\begin{array}{l}\text { № } \\
\text { Ítem }\end{array}$} & \multirow{2}{*}{ Reactivos } & \multicolumn{2}{|c|}{ Componentes } \\
\hline & & 1 & 2 \\
\hline 75 & He pasado periodos en los que he fumado marihuana varias veces a la semana. & 0.79 & \\
\hline 40 & Solía quedar tan drogado(a) que no sabía lo que estaba haciendo. & 0.77 & \\
\hline 111 & He tenido algunos "encuentros" con la ley. & 0.71 & \\
\hline $8^{*}$ & Pase lo que pase, nunca consumiría drogas. & 0.62 & \\
\hline 120 & Ha habido veces en las que no he podido pasar el día sin un poco de marihuana. & 0.60 & \\
\hline 150 & Frecuentemente me divierto haciendo ciertas cosas que son ilegales. & 0.48 & 0.36 \\
\hline 42 & Veo que estoy muy lejos de lo que en realidad me gustaría ser. & 0.45 & \\
\hline $45^{*}$ & Nunca he sido llamado(a) "delincuente juvenil". & 0.43 & \\
\hline 21 & El castigo nunca me detuvo para hacer lo que yo quería. & 0.34 & 0.33 \\
\hline 128 & No me importa pasar por sobre otras personas para demostrar mi poder. & & 0.69 \\
\hline 52 & No veo nada malo en usar a otros para obtener lo que quiero. & & 0.61 \\
\hline 92 & Soy muy bueno(a) inventando excusas para salir de los problemas. & & 0.57 \\
\hline 28 & A veces asusto a otros chicos para que hagan lo que yo quiero. & & 0.56 \\
\hline 117 & Hago lo que quiero sin preocuparme si afecta a los otros. & & 0.55 \\
\hline 135 & Puedo "encantar" a la gente para que me den casi todo lo que yo quiero. & & 0.47 \\
\hline $5^{*}$ & Hago lo mejor que puedo para no herir los sentimientos de los demás. & & 0.44 \\
\hline 152 & Cuando estamos divirtiéndonos, mis amigos y yo podemos quedar bastante borrachos. & 0.37 & 0.41 \\
\hline $23 *$ & Me gusta seguir instrucciones y hacer lo que otros esperan de mí. & & 0.36 \\
\hline $9 *$ & Siempre trato de hacer lo que es correcto. & & 0.34 \\
\hline \multirow[t]{4}{*}{62} & Disfruto pensando en sexo. & & \\
\hline & Autovalores & 5.05 & 1.77 \\
\hline & Porcentaje de la varianza explicada & 25.25 & 8.85 \\
\hline & Coeficiente Alfa & 0.81 & 0.76 \\
\hline
\end{tabular}

Nota. Los reactivos han sido tomados de la versión chilena del MACI (Vinet et al., 1999).

* Ítem de puntuación invertida.

Fuente: elaboración propia. 
Escala de Psicopatía de 16 ítems (P-16)

El análisis factorial de esta escala, utilizando el método de extracción por componentes principales con rotación Oblimin, entrega una estructura libre de cuatro factores con autovalores mayores a 1.0 que explica el $44.8 \%$ de la varianza, pero que es de difícil interpretación teórica. Al forzar el análisis a tres factores, tal como lo hicieran Penney et al. (2008), se explica el $38.5 \%$ de la varianza y se obtiene una estructura clara, donde todos los elementos presentan cargas factoriales mayores a 0.30 , esta estructura es presentada en la Tabla 3. A través del contenido de los ítems se puede apreciar que el primer componente correspondería a la dimensión Conducta Antisocial, el segundo a la dimensión Dureza o Insensibilidad Emocional y el tercero a la dimensión Egocentrismo planteados en el modelo de Cooke y Michie (2001). Las correlaciones entre los factores son de mayor a menor: F1 у F3: $r=0.21$; F1 у F2: $r=0.19 ;$ F2 у F3: $r=0.12$.

\section{TABLA 3}

Estructura factorial de la escala Psicopatía - 16 ítems en adolescentes chilenos

\begin{tabular}{|c|c|c|c|c|}
\hline \multirow{2}{*}{$\begin{array}{l}\text { № } \\
\text { Ítem }\end{array}$} & \multirow{2}{*}{ Reactivos } & \multicolumn{3}{|c|}{ Componentes } \\
\hline & & 1 & 2 & 3 \\
\hline $15^{*}$ & Nunca he hecho algo por lo cual me pudieran haber arrestado. & 0.79 & & \\
\hline 111 & He tenido algunos "encuentros" con la ley. & 0.78 & & \\
\hline $45^{*}$ & Nunca he sido llamado(a) "delincuente juvenil". & 0.61 & & \\
\hline 150 & Frecuentemente me divierto haciendo ciertas cosas que son ilegales. & 0.54 & & \\
\hline 21 & El castigo nunca me detuvo para hacer lo que yo quería. & 0.41 & & \\
\hline 155 & Decir mentiras es una cosa muy normal. & 0.32 & & \\
\hline $23 *$ & Me gusta seguir instrucciones y hacer lo que otros esperan de mí. & 0.29 & & \\
\hline 61 & Al parecer no tengo muchos sentimientos por los demás. & & 0.71 & \\
\hline 60 & No me molesta ver a alguien sufriendo. & & 0.68 & \\
\hline 52 & No veo nada malo en usar a otros para obtener lo que quiero. & & 0.54 & 0.33 \\
\hline $5 *$ & Hago lo mejor que puedo para no herir los sentimientos de los demás. & & 0.48 & \\
\hline 139 & Yo me burlaría de alguien en un grupo solo para humillarlo(a). & & 0.42 & 0.30 \\
\hline 103 & Me gusta ser el centro de atención. & & & 0.68 \\
\hline 146 & En muchas cosas me siento muy superior a la mayoría de la gente. & & & 0.64 \\
\hline 135 & Puedo "encantar" a la gente para que me den casi todo lo que yo quiero. & & & 0.57 \\
\hline 7 & Algunas personas piensan que soy un poco presumido(a). & & & 0.55 \\
\hline & Autovalores & 2.98 & 1.65 & 1.52 \\
\hline & Porcentaje de la varianza explicada & 18.64 & 10.34 & 9.49 \\
\hline & Coeficiente Alfa & 0.64 & 0.58 & 0.58 \\
\hline
\end{tabular}

Nota. Los reactivos provienen de la versión chilena del MACI (Vinet et al., 1999).

* Ítem de puntuación invertida.

Fuente: elaboración propia. 
Identificación y descripción de los

adolescentes con alta psicopatía

Los estadísticos descriptivos de las escalas factoriales en cada uno de los grupos y la contrastación entre grupos, se presentan en la Tabla 4. Se puede observar que hay un incremento lineal a través de los grupos en la puntuación de las dimensiones que evalúan conducta antisocial en las dos escalas de psicopatía (Factor 1, en cada escala); sin embargo, en las dimensiones que evalúan aspectos de personalidad (Factor 2, en ambas escalas), este incremento lineal se rompe pues los puntajes medios mayores son compartidos por los escolares que autorreportan alta trasgresión (E-AT) y los infractores reincidentes (AI-RE), es destacable que el mayor puntaje medio en Dureza/Insensibilidad se presenta en el grupo E-AT. El Factor 3 de la escala P-16 muestra que el grupo E-BT se diferencia significativamente de los otros tres grupos, mostrando un menor nivel de egocentrismo.

Estos resultados sugieren que los jóvenes con un nivel alto de psicopatía deberían presentar características de conducta antisocial alta y una elevación de las características afectivas e interpersonales; estas condiciones son representadas en el siguiente algoritmo generado para identificar a los jóvenes con alto nivel de psicopatía:

$$
\begin{aligned}
& \text { Factor } 1 \text { de la PCS }>M_{\text {TOTAL }}+1 \text { DT o } \\
& \text { Factor } 1 \text { de la } \mathrm{P}-16>\mathrm{M}_{\text {TOTAL }}+1 \mathrm{DT} \text { y } \\
& \text { Factor } 2 \text { de la PCS }>M_{(E-A T+A I-R E)}+1 D T \text { y } \\
& \text { Factor } 2 \text { de la P-16 }>\mathrm{M}_{(\mathrm{E}-\mathrm{AT}+\mathrm{AI}-\mathrm{RE})}+1 \mathrm{DT}
\end{aligned}
$$

Los adolescentes seleccionados a través de este algoritmo cumplen con siguientes condiciones: [(F1 de la PCS $\geq 8)$ o (F1 de la P-16 $\geq 6)]$ y [(F2 de la PCS $\geq 6)$ y (F2 de P-16 $\geq 2$ )]. Son ellos, 49 jóvenes (11.2 \% de la muestra total), con una edad media de 17.5 años $(D T=1.12)$ y una escolaridad media de 9.04 años de educación formal (DT $=$ 2.08). Se distribuyen entre los grupos E-AT (14 sujetos, $11.4 \%$ del grupo), AI-IN (8 sujetos, $8.4 \%$ del grupo) y AI-RE (27 sujetos, $28.7 \%$ del grupo). Las características de su conducta social (CACSA) se presentan en la Tabla 5, donde se observa que la única escala en la cual este grupo no se diferencia significativamente del resto de la muestra es Comportamiento Prosocial $(t=1.838 ; p=0.067)$.

TABLA 4

Estadísticos descriptivos y comparación grupal en las escalas factoriales de Psicopatía

\begin{tabular}{lcccccccccc}
\hline \multirow{2}{*}{ Escalas Factoriales } & \multicolumn{2}{c}{ E-BT } & \multicolumn{2}{c}{ E-AT } & \multicolumn{2}{c}{ AI-IN } & \multicolumn{2}{c}{ AI-RE } & \multirow{2}{*}{$F$} \\
\cline { 2 - 9 } & $\mathrm{M}$ & DT & $\mathrm{M}$ & $\mathrm{DT}$ & $\mathrm{M}$ & $\mathrm{DT}$ & $\mathrm{M}$ & $\mathrm{DT}$ & \\
\hline De la PCS & & & & & & & & & \\
F1 Conducta antisocial/drogas & $1.48_{\mathrm{a}}$ & 1.25 & $4.76_{\mathrm{b}}$ & 2.09 & $5.31_{\mathrm{b}}$ & 2.60 & $7.65_{\mathrm{c}}$ & 1.81 & $184.53^{* * *}$ \\
F2 Afectivo/interpersonal & $2.11_{\mathrm{a}}$ & 1.68 & $5.86_{\mathrm{b}}$ & 2.42 & $4.57_{\mathrm{c}}$ & 2.75 & $5.96_{\mathrm{b}}$ & 3.01 & $62.48^{* * * *}$ \\
De la P-16 & & & & & & & & & \\
F1 Conducta antisocial & $1.78_{\mathrm{a}}$ & 1.21 & $3.92_{\mathrm{b}}$ & 1.63 & $4.65_{\mathrm{c}}$ & 1.51 & $5.20_{\mathrm{d}}$ & 1.37 & $122.43^{* * *}$ \\
F2 Dureza/insensibilidad & $0.65_{\mathrm{a}}$ & 0.96 & $1.69_{\mathrm{b}}$ & 1.39 & $1.08_{\mathrm{c}}$ & 1.15 & $1.34_{\mathrm{bc}}$ & 1.40 & $15.40^{* * * *}$ \\
F3 Egocentrismo & $1.31_{\mathrm{a}}$ & 1.16 & $2.46_{\mathrm{b}}$ & 1.52 & $1.96_{\mathrm{b}}$ & 1.52 & $2.43_{\mathrm{b}}$ & 1.75 & $15.47^{* * * *}$ \\
\hline
\end{tabular}

Nota. E-BT: Escolares con baja trasgresión autorreportada; E-AT: Escolares con alta trasgresión autorreportada; AI-IN: Adolescentes infractores de inicio; AI-RE: Adolescentes infractores reincidentes. Los grupos que tienen el mismo subíndice no presentan diferencias significativas entre sí.

$* * * p<0.001$

Fuente: elaboración propia. 
TABLA 5

Estadísticos descriptivos del CACSA en los adolescentes con alta psicopatía

\begin{tabular}{|c|c|c|c|c|c|}
\hline \multirow[t]{2}{*}{ Escalas CACSA } & \multicolumn{2}{|c|}{$\begin{array}{c}\text { Alta } \\
\text { Psicopatía } \\
n=49\end{array}$} & \multicolumn{2}{|c|}{$\begin{array}{c}\text { Resto } \\
\text { Muestra } \\
n=387\end{array}$} & \multirow[t]{2}{*}{$\begin{array}{c}\text { Prueba } \\
t\end{array}$} \\
\hline & $M$ & DT & M & DT & \\
\hline $\begin{array}{l}\text { Comportamiento } \\
\text { antisocial total }\end{array}$ & 64.94 & 27.96 & 28.93 & 25.59 & $9.18 * * *$ \\
\hline $\begin{array}{l}\text { Trasgresiones } \\
\text { sociales leves }\end{array}$ & 21.00 & 9.21 & 11.21 & 8.89 & $7.24 * * *$ \\
\hline $\begin{array}{l}\text { Trasgresiones con- } \\
\text { tra las personas }\end{array}$ & 18.51 & 11.42 & 7.56 & 8.37 & $6.49 * * *$ \\
\hline $\begin{array}{l}\text { Trasgresiones con- } \\
\text { tra la propiedad }\end{array}$ & 13.43 & 6.96 & 5.48 & 6.23 & $8.30 * * *$ \\
\hline Delitos & 20.49 & 10.43 & 8.65 & 9.12 & $8.42 * * *$ \\
\hline $\begin{array}{l}\text { Consumo de } \\
\text { drogas }\end{array}$ & 17.06 & 8.20 & 6.85 & 7.52 & $8.86 * * *$ \\
\hline $\begin{array}{l}\text { Trasgresión de } \\
\text { normas para la } \\
\text { edad }\end{array}$ & 15.53 & 8.09 & 7.72 & 7.42 & $6.88 * * *$ \\
\hline $\begin{array}{l}\text { Comportamiento } \\
\text { prosocial }\end{array}$ & 26.04 & 12.74 & 29.59 & 12.73 & -1.84 \\
\hline
\end{tabular}

Fuente: elaboración propia.
Las características de personalidad del grupo se presentan en la Tabla 6. Allí se muestran las puntuaciones en las escalas clínicas del MACI del grupo expresadas en puntajes transformados con puntos de corte (Vinet \& Forns, 2008) y se comparan con las puntuaciones del resto de la muestra, incluyendo el valor eta ${ }^{2}$ para apreciar la proporción de la variabilidad total de cada escala clínica que se explica por la pertenencia a cada grupo, facilitando de este modo la interpretación del funcionamiento psicológico de los jóvenes psicópatas.

La interpretación psicológica que se desarrolla a continuación, utiliza la definición de las escalas según Millon (1993), las interpretaciones configuracionales de McCann (1999), los hallazgos culturales planteados por Vinet (2010) para sujetos latinoamericanos y la propuesta chilena de normas para el MACI (Vinet \& Forns, 2008). A partir de estos elementos, se describen las características siguientes en los adolescentes psicópatas.

Al igual que el resto de los adolescentes, muestran una aceptable capacidad para experimentar placer y establecer vínculos sociales (escala Afligido), privilegiando sus necesidades individuales y mostrando cierta dificultad para postergar sus gratificaciones (escalas Dramatizador y Egoísta); han asumido favorablemente sus cambios corporales (Desaprobación Corporal), la interacción

TABLA 6

Estadísticos descriptivos del MACI en los adolescentes con alta psicopatía

\begin{tabular}{|c|c|c|c|c|c|c|c|c|}
\hline & Escalas MACI & $\begin{array}{r}\text { Alta } \\
n\end{array}$ & $\begin{array}{l}\text { opatía } \\
99\end{array}$ & $\begin{array}{r}\text { Rest } \\
n\end{array}$ & $\begin{array}{l}\text { lestra } \\
87\end{array}$ & Prue & & eta $^{2}$ \\
\hline 1 & Introvertido & 56.88 & 12.77 & 47.37 & 14.75 & 4.31 & $* * *$ & 0.04 \\
\hline $2 \mathrm{~A}$ & Inhibido & 50.63 & 17.31 & 48.29 & 16.13 & 0.95 & & 0.00 \\
\hline $2 \mathrm{~B}$ & Afligido & 69.67 & 14.62 & 49.30 & 21.24 & 8.67 & $* * *$ & 0.09 \\
\hline 3 & Sumiso & 67.43 & 10.38 & 51.77 & 11.14 & 9.86 & $* * *$ & 0.17 \\
\hline 4 & Dramatizador & 48.33 & 13.44 & 49.62 & 14.69 & 0.59 & & 0.00 \\
\hline 5 & Egoísta & 44.69 & 14.52 & 49.32 & 14.09 & -2.16 & $*$ & 0.01 \\
\hline $6 \mathrm{~A}$ & Trasgresor & 79.51 & 8.36 & 54.13 & 15.79 & 17.64 & $* * *$ & 0.22 \\
\hline $6 \mathrm{~B}$ & Poderoso & 77.31 & 11.53 & 48.86 & 20.03 & 14.69 & $* * *$ & 0.18 \\
\hline
\end{tabular}




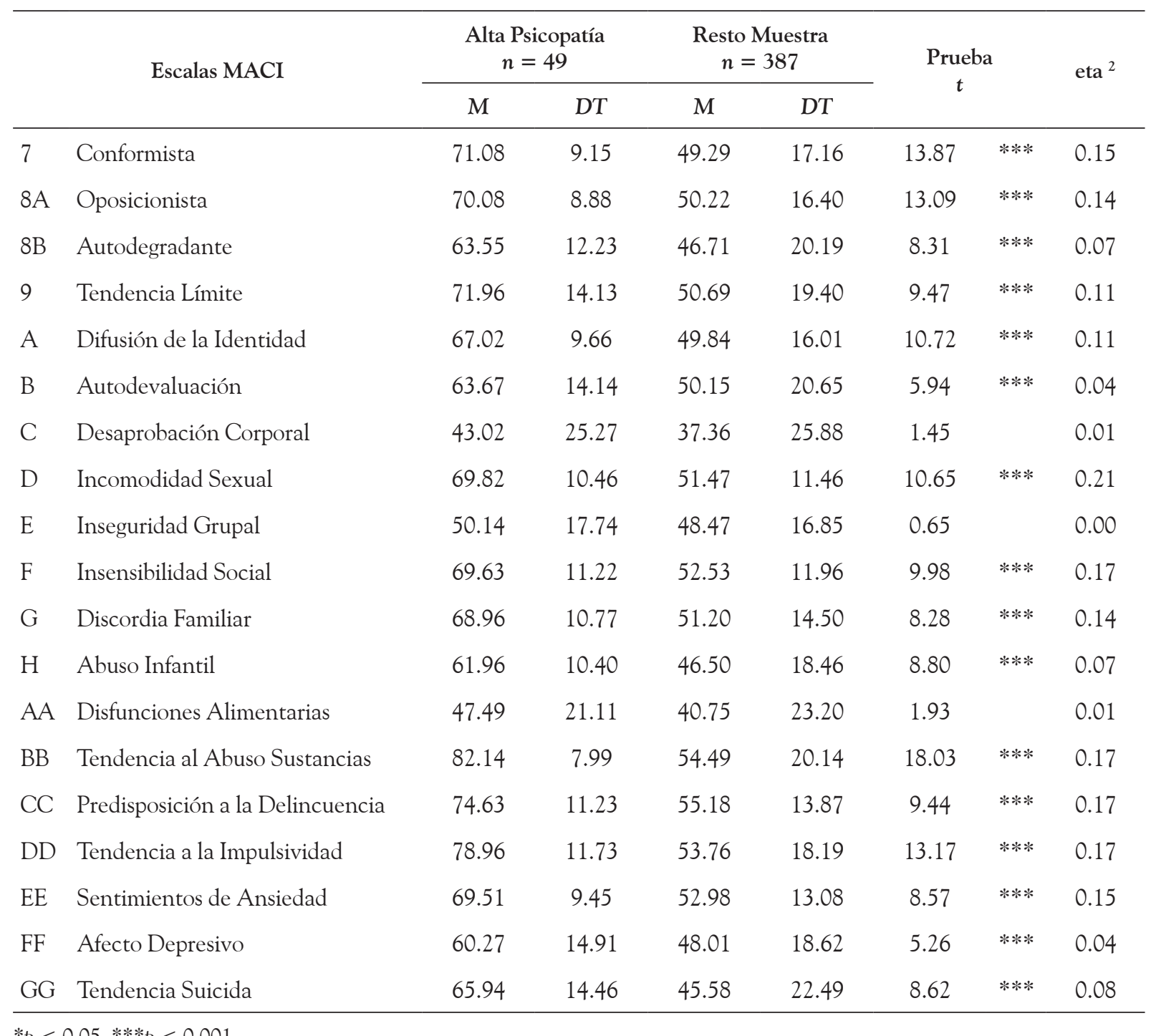

$* p<0.05, * * * p<0.001$

Fuente: elaboración propia.

social no les causa malestar ni incomodidad en sus vidas (Inseguridad Grupal) y, como la gran mayoría de los varones, no presentan trastornos alimentarios (Disfunciones Alimentarias). En lo particular, los adolescentes con alta psicopatía se caracterizan por un estilo de funcionamiento ostensiblemente trasgresor y despiadado (escalas Trasgresor y Poderoso), con incapacidad para sentir culpa o remordimiento genuinos; manifiestan conductas de rebeldía y constante desafío a las normas establecidas (escalas Sumiso y Conformista) y son pleitistas y pendencieros con sus pares, y desafiantes y provocadores con los adultos (Opo- sicionista); destaca su liberalidad en la sexualidad, presentando desinhibición sexual, precocidad y/o conductas promiscuas (Incomodidad Sexual); presentan frialdad e indiferencia por los sentimientos y el bienestar de los demás, apareciendo como fríos y crueles, o bien manipuladores y superficialmente cordiales (Insensibilidad Social); su perfil da cuenta de un alto grado de tensión y conflicto con sus padres, percibiendo las relaciones familiares como forzadas y no apoyadoras (Discordia Familiar y Abuso Infantil). La patología más prevalente está representada por la tríada formada por el abuso de sustancias, la delincuencia y la impulsividad 
(escalas de Tendencia al Abuso de Sustancias, Predisposición a la Delincuencia y Tendencia a la Impulsividad) acompañada de escasa ansiedad (escala de Sentimientos de Ansiedad).

\section{Discusión}

El abordaje del constructo de psicopatía infanto-juvenil, a través de una evaluación multidimensional desarrollada con el MACI, ha permitido comprender mejor los diferentes elementos implicados en este constructo, al aplicarlo a un grupo de adolescentes representantes del continuo adaptación social-desadaptación social. Esta discusión evalúa, primeramente, las características psicométricas de las escalas de Psicopatía del MACI; en segundo lugar, aborda las características de la psicopatía infanto-juvenil tal como se observan a través de los cuatro grupos estudiados; por último, se refiere a la descripción de las características del grupo con alta psicopatía y su utilidad en la evaluación de adolescentes con problemas de desadaptación social.

Con respecto a las escalas de Psicopatía del MACI, uno de los primeros aspectos que resalta en el estudio es la correspondencia que los ítems de las escalas poseen con respecto a los dos modelos de psicopatía actualmente vigentes. El modelo de Hare (2003) es representado claramente en la estructura de dos factores del PCS, donde se observa la preeminencia de las conductas trasgresoras, entre las que se incluye el abuso de alcohol y drogas, por sobre las características afectivas/interpersonales de la psicopatía. Por su parte, el modelo de Cookie y Mitchie (2001) es representado por los 16 ítems del P-16, a través de un ordenamiento en tres factores -Conducta Antisocial, Dureza o Insensibilidad Emocional y Egocentrismo- que aparecen sin ambigüedades. Además, en este análisis hay que destacar la mejor consistencia interna de estas escalas factoriales que la consistencia de las facetas de contenido derivadas conceptualmente por Salekin et al. (2003). Los niveles de consistencia interna determinados para las escalas factoriales, avalan su uso en diversos grupos de adolescentes.

La descripción de los cuatro grupos de la muestra de estudio presenta evidencias sobre el aumento progresivo de las conductas trasgresoras, según el ordenamiento de los grupos en el continuo adaptación-desadaptación social (escolares con baja trasgresión, escolares con alta trasgresión, adolescentes infractores de inicio, adolescentes infractores reincidentes). Este incremento de conductas trasgresoras ha sido objetivado a través del CACSA y de los aspectos conductuales de las escalas de psicopatía. Sin embargo, llama la atención que ese ordenamiento se altera cuando se trata de características de personalidad; en este caso, las puntuaciones altas obtenidas por el grupo de infractores reincidentes (ubicados al final del continuo) son compartidas por los escolares que reportan alta trasgresión (ubicados en una posición intermedia), quienes no han sido visibilizados por los sistemas judiciales como infractores de ley. La presencia de características psicopáticas en escolares que autorreportan alta trasgresión pero que no presentan conflictos con los sistemas judiciales, es un antecedente para considerar la relevancia de factores situacionales, familiares y comunitarios en la manifestación de la conducta trasgresora, pues implica que solo algunos jóvenes con características psicopáticas son visualizados por los sistemas judiciales y calificados como delincuentes o infractores de ley en razón de sus delitos.

Al respecto, la teoría señala que los factores externos a la propia persona, juegan un rol relevante en la manifestación de los comportamientos delincuenciales a través de las "trayectorias delictivas" (Piquero et al., 2007); sin embargo, la determinación empírica del peso específico de los elementos que van determinando las trayectorias no ha sido lograda totalmente, señalándose, de modo amplio, que en la trayectorias delictivas, además de los factores personales, juegan un rol determinante los entornos de alto riesgo criminogénico caracterizados por un medio familiar alterado (generalmente con comportamientos abusivos hacia los jóvenes), escasa supervisión de los padres, presencia de pares o amigos con comportamiento delictivo, alto consumo y/o abuso de alcohol y drogas (Le Blanc \& Fréchette, 1989). Por otro lado, tampoco se ha determinado fehacientemente qué elementos ac- 
túan como factores protectores, cuando hay una alta disposición a la psicopatía que podría conducir a la delincuencia. Dado que estas condiciones ameritan ser investigadas en profundidad con objetivos de prevención y de desarrollo de programas de intervención para la conducta delictiva adolescente, el presente estudio hace un aporte significativo, pues el algoritmo desarrollado a partir de las puntuaciones en las escalas de psicopatía constituye una herramienta para pesquisar a aquellos jóvenes que presentan características psicopáticas, independientemente de su condición de infractores de ley o no. La identificación del grupo con alta psicopatía, a través del algoritmo desarrollado en este estudio, permitiría un estudio en profundidad considerando, junto a las características de personalidad, los elementos del entorno y situacionales, que pueden hacer la diferencia entre trayectorias delictuales que se afianzan en el tiempo y otras que se debilitan y desaparecen.

Los adolescentes pesquisados al aplicar este algoritmo presentan un perfil de personalidad que reúne muchas de las características asociadas a la psicopatía en la adultez. Ellas son descritas a través de la interpretación de las escalas del MACI en las diferentes áreas de funcionamiento que cubre este instrumento. Así, se describe un estilo de funcionamiento estable trasgresor y despiadado, con conductas de rebeldía y oposicionismo (patrones de la personalidad), que contextualmente presenta características de desinhibición sexual, frialdad y/o manipulación interpersonal y conflictos familiares (preocupaciones expresadas), y que va asociado a trastornos por abuso de sustancias, conductas impulsivas y delincuenciales con nula o escasa ansiedad (síndromes clínicos). Este perfil se manifiesta en aproximadamente un tercio de los adolescentes infractores (28.2\% de los reincidentes y $8.4 \%$ de los infractores de inicio), pero también en más del $10 \%$ de los escolares que autorreportan alta trasgresión. Esta diversidad de jóvenes indicaría que, aunque la condición de alta psicopatía es prevalente en los grupos de infractores de ley, también se presenta en los adolescentes no infractores; estos últimos constituyen un grupo de interés para profundizar en el estudio de las condiciones per- sonales y del entorno ecológico de los jóvenes que posibilitan o no la conducta delictual.

Las limitaciones de este estudio están dadas por el abordaje de un tema polémico, complejo y sobre el cual existen escasos reportes en América Latina y pocos instrumentos que permitan objetivarlo adecuadamente. Este estudio espera haber realizado un aporte al profundizar el conocimiento, en un contexto sociocultural latinoamericano, de las escalas de Psicopatía del MACI, las cuales se presentan como una herramienta promisoria para la detección de características psicopáticas en adolescentes.

\section{Referencias}

Alarcón, P., Pérez-Luco, R., Salvo, S., Roa, G., Jaramillo, K. \& Sanhueza, C. (2010). Validación del Cuestionario de auto-reporte de conducta antisocial y prosocial en adolescentes: CACSA. Paideia, 20, 47, 291-302.

Alarcón, P., Vinet, E. \& Salvo, S. (2005). Estilos de personalidad y desadaptación social durante la adolescencia. Psykhe, 14 (1), 3-16.

Alarcón, P., Vinet, E., Salvo, S. \& Pérez-Luco, R. (2009). Caracterización y evaluación multidimensional de adolescentes con desadaptación social (Informe Final. Proyecto Fondecyt 1070397). Santiago: CONICYT, Ministerio de Educación de Chile.

Cooke, D. J. \& Michie, C. (2001). Refining the construct of psychopathy: Towards a hierarchical model. Psychological Assessment, 13, 171-188.

Farrington, D. (1996). The explanation and prevention of youthful offending. In P. Cordelia \& L. Siegel (Eds.), Readings in contemporary criminological theory (pp. 257-272). Boston: Northeastern University Press.

Forth, A. E., Kosson, D. S. \& Hare, R. D. (2003). Psychopathy Checklist-Youth Version: Technical Manual. Toronto: Multi-Health Systems.

Frick, P. J., Barry, C. T. \& Bodin, S. D. (2000). Applying the concept of psychopathy to children: Implications for the assessment of antisocial youth. In C. B. Gacono (Ed.), The clinical and forensic assessment of psychopathy: A practitioner's guide (pp. 3-24). Mahwah, NJ: Erlbaum. 
Frick, P. J. \& Ellis, M. (1999). Callous-unemotional traits and subtypes of conduct disorder. Clinical Child and Family Psychology Review, 2, 149-168.

Frick, P. J., O’Brien, B. S., Wootton, J. M. \& McBurnett, K. (1994). Psychopathy and conduct problems in children. Journal of Abnormal Psychology, 103, 700-707.

Hare, R. D. (2003). Psychopathy Checklist Revised (PCLR) (2nd ed.). Toronto: Multi- Health Systems.

Le Blanc, M. \& Fréchette, M. (1989). Male criminal activity from childhood through youth. Berlin/New York: Springer-Verlag.

Lynam, D. R. (1996). The early identification of chronic offenders: Who is the fledgling psychopath? Psychological Bulletin, 120, 209-234.

McCann, J. (1999). Assessing adolescents with the MACI. Using the Millon Adolescent Clinical Inventory. New York: Wiley \& Sons.

Millon, T. (1969). Modern psychopathology: A biosocial approach to maladaptative learning and functioning. Philadelphia: Saunders.

Millon, T. (1990). Toward a new personology. An evolutionary model. New York: Wiley \& Sons.

Millon, T. (1993). Manual of Millon Adolescent Clinical Inventory. Minneapolis: Nacional Computer Systems.

Moffitt, T. E. (1993). Adolescence-limited and lifecourse persistent antisocial behavior: A developmental taxonomy. Psychological Review, 100, 674-701.

Moffitt, T. E., Caspi, A., Harrington, H. \& Milne, B. J. (2002). Males on the life-course-persistent and adolescence-limited antisocial pathways. Followup at age 26 years. Development $\mathrm{v}$ Psychopathology, 14, 179-207.

Murrie, D. C. \& Cornell, D. G. (2000). The Millon Adolescent Clinical Inventory and psychopathy. Journal of Personality Assessment, 75 (1), 110-125.

Penney, S. R, Moretti, M. M. \& Da Silva, K. S. (2008). Structural validity of the MACI Psychopathy and Narcisism scales: Evidence of multidimensionality and implications for the use in research and screening. Journal of Clinical Child \& Adolescent Psychology, 37 (2), 422-433.

Piquero, A. R., Farrington, D. P. \& Blumstein, A. (2007). Key issues in criminal career research. New analyses of the Cambridge study in delinquent development. Cambridge, UK: Cambridge University Press.

Romero, E. (2001). El constructo psicopatía en la infancia y la adolescencia: del trastorno de conducta a la personalidad antisocial. Anuario de Psicología, 32, 25-49.

Rutter, M., Giller, H. \& Hagell, A. (2000). La conducta antisocial de los jóvenes. Madrid: Cambridge University Press.

Salekin, R. T., Ziegler, T., Larrea, M., Anthony, V. \& Bennett, A. (2003). Predicting dangerousness with two Millon Adolescent Clinical Inventory Psychopathy scales: The importance of egocentric and callous traits. Journal of Personality Assessment, 80 (2), 154-163.

Vinet E. (2010). Relativismo Cultural del Modelo de Personalidad de Millon en América Latina: Un estudio con adolescentes. Interdisciplinaria 27,1, 23-40.

Vinet, E. \& Alarcón, P. (2003). El Inventario Clínico para Adolescentes de Millon (MACI) en la evaluación de adolescentes chilenos. Psykhe, 12 (1), 39-55.

Vinet, E. \& Alarcón, P. (2009). Caracterización de personalidad de mujeres adolescentes infractoras de ley: un estudio comparativo. Paideia, 19 (43), 143-152.

Vinet, E., Brió, C., Correa, P., Díaz, P., Diez, M., Echeverría, M., Salazar, D. \& Vargas, A. (1999). MACI. Traducción y adaptación chilena para uso exclusivo en investigación (Proyecto DIDUFRO 9966). Temuco, Chile: Universidad de La Frontera.

Vinet, E. \& Forns, M. (2008). Normas chilenas para el MACI: una integración de criterios categoriales y dimensionales. Terapia Psicológica, 26 (2), 151-163.

Zúñiga, D., Vinet, E., y León, E. (2011). Caracterización psicométrica del Psychopathy Checklist: Youth Version (PCL:YV) en adolescentes chilenos. Terapia Psicológica, 29, 1, 25-31. 
